\title{
DEVICE TEMPORAL FORENSICS: AN INFORMATION THEORETIC APPROACH
}

\author{
Junwen Mao, Orhan Bulan, Gaurav Sharma, Suprakash Datta* \\ ECE Dept., University of Rochester, Rochester, NY 14627-0126 \\ ${ }^{*}$ CSE Dept., York University, Toronto, Canada
}

\begin{abstract}
By formulating the problem of ordering the outputs observed from a device over time, we pose a new problem in forensics and propose a framework for addressing this problem of device temporal forensics. Our proposed framework is based on a two-stage approach wherein time-dependent device parameters are first estimated from observed outputs and the resulting estimates are then temporally ordered by employing a Markov model for the temporal evolution of device parameters and exploiting the data processing inequality in information theory. We demonstrate and evaluate a simple realization of the framework for digital camera forensics based on photo-response non-uniformity. Results obtained over a database of online images indicate that the method provides accurate temporal ordering.
\end{abstract}

Index Terms - Device temporal forensics, temporal ordering, PRNU(photo-response nonuniformity noise)

\section{INTRODUCTION}

Device forensics represents an important class of image forensic techniques that have received considerable attention in recent research. The objective of device forensics is to reliably identify the imaging device associated with a given image signal. Specific sub-goals within this larger objective, depending on the application scenario, have included identification of the digital camera or scanner that was used to capture a digital image $[1,2,3]$ or identification of a printer utilized for printing a given document $[4,5]$.

In a number of image forensic applications, it is not sufficient to identify the imaging device alone - and additional information is desirable. Temporal localization of the image and device linkage, in particular, can be crucial ${ }^{1}$. For instance, consider a situation where a digital camera found to be associated with illicit content distributed over the Internet has also changed ownership. In this situation, temporal localization of the camera image association, i.e. an estimate of the time when the images with illicit content were captured, can not only help to improve the "prosecutorial strength" of the evidence but can also allow for a more focused and effective investigation. In a similar fashion, temporal localization can also be critical for establishing the validity of printed legal documents - for instance, by determining whether a will was printed before or after the expiration of the purported testator.

Motivated by the importance of temporal localization, in this paper, we propose a framework for device temporal forensics. A number of device forensic techniques rely on estimation of parameters of the imaging device $[1,2,4,5]$. By suitably modeling the temporal

This work was supported in part by Air Force Office of Scientific Research (AFOSR) and by a grant from New York State Office of Science, Technology and Academic Research (NYSTAR) through the Center for Electronic Imaging Systems (CEIS).

${ }^{1}$ Temporal information also plays a vital role for forensic investigations in domains other than digital image forensics $[6,7]$.

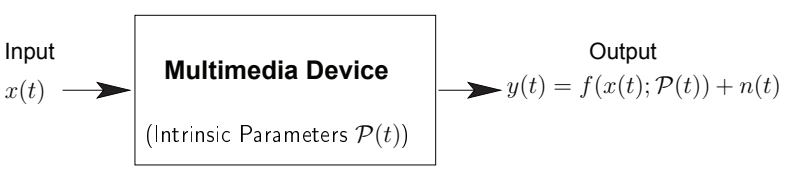

Fig. 1. Multimedia recording device with temporally evolving parameters

evolution of the device parameters, our framework augments several of these forensic methods to additionally provide time-ordering information. Specifically, in our framework we utilize a Markov model for the evolution of device parameters which then allows us to obtain estimates of temporal ordering by exploiting the data processing inequality [8, pp. 34] in information theory. Due to space constraints, we demonstrate only one practical realization of the framework and defer additional applications that are under development to future work. Specifically, here we augment the digital camera forensic technique of [1] that relies on estimation of photo-response non-uniformity (PRNU) parameters to obtain temporal ordering capability based on framework.

In Section 2, we provide a model for temporal evolution in multimedia devices. In Section 3, we outline our framework and propose an efficient algorithm for temporal ordering based on the data processing inequality. Section 4 outlines a specific realization of the framework for the PRNU based temporal forensics for digital cameras. Experimental results for this realization are presented in Section 5. Concluding remarks and a discussion form the final Section 6.

\section{TEMPORAL EVOLUTION OF MULTIMEDIA DEVICE PARAMETERS}

In order to capture the temporal characteristics of a multimedia device, we model the input-output relation for the device using the generic model illustrated in Fig. 1. At a given time instant $t$, the device output $y(t)$ is modeled as

$$
y(t)=f(x(t) ; \mathcal{P}(t))+n(t),
$$

where $x(t)$ denotes the input, $\mathcal{P}(t)$ the intrinsic parameters for the device, and $n(t)$ denotes non systematic noise that is independent across different device outputs. For imaging devices, for each time instant $t, x(t), y(t)$ and $n(t)$ are two-dimensional signals and in general the device intrinsic parameters $\mathcal{P}(t)$ also constitute a vectorvalued and potentially multi-dimensional signal. In general, the parameters $\mathcal{P}(t)$ may represent only a partial subset of the full parameter space that characterizes the device operation.

The model outlined above is implicit in a number of device forensic methods, albeit without the temporal dependence for the intrinsic device parameters. The typical operation of these methods can be summarized as follows: First, an estimate of the device 
parameters is obtained from outputs known to be originated by the device. An image under investigation is then associated with a specific device by determining whether the image is consistent with the parameters estimated for the specific device. For practical deployment of this methodology, systematic device degradations in particular have proven to be useful device parameters for enabling image forensics: Among other examples, photo-response non-uniformity (PRNU) [9, 1] and sensor dust [10] have been exploited for digital camera forensics, noise features for scanner forensics $[11,2]$, and printer geometric distortion for printer identification [5]. Incorporating, temporal dependence in these intrinsic device parameters allows us to comprehend their temporal evolution within our model, which forms the basis of our temporal forensics formulation.

As a specific case of our general model (1), we consider digital camera forensics based on PRNU estimation. For this scenario, by adding time dependence to the sensor PRNU representation of $[9,1]$ we obtain the following model. At time instant $t$, the image $I(t)$ captured by the camera is given by

$$
I(t)=I^{(0)}(t) \cdot(1+K(t))+\Theta(t)
$$

where $I^{(0)}(t)$ represents the ideal "input" image at the camera sensor that is free from sensor PRNU variations, $K(t)$ represents the timedependent PRNU parameters for the digital camera, $\cdot$ represents the element-by-element multiplication operation, and $\Theta(t)$ represents non-systematic noise, which is statistically independent for different images captured by the camera. Through the rest of this paper we will use this particular problem and model for illustrating our methodology.

\section{DATA PROCESSING INEQUALITY FOR TEMPORAL ORDERING}

We consider a two stage process for temporal ordering wherein first the parameters $\mathcal{P}(t)$ are estimated using device outputs and then a temporal ordering is determined for these estimated parameters. If a parametric model is available for the temporal evolution of the device parameters, it can be utilized to establish the temporal ordering. Invariably, however, such a model is unavailable and we therefore propose a non-parametric formulation. Specifically, we consider scenarios where the device parameter evolution can be modeled as a Markov Process. As indicated earlier, in a number of situations, the device parameters represent systematic device degradations such as camera sensor PRNU or dust, printer geometric distortions, etc. If the systematic degradations are the accumulation of independent individual degradations, the resulting overall degradation can be modeled as an independent increments process, which forms a Markov process [12, pp. 501]. Camera sensor pixel failures and sensor dust specks, for instance, can be readily modeled in this fashion. It should also be clear that some device parameters, for example the color filter array pattern, are temporally invariant. While these may provide useful forensic information, they are ineffective for temporal forensics.

If $\mathcal{P}(t)$ is a Markov Process then for any $L$ monotonically increasing time instants $t_{1}<t_{2}<\ldots<t_{L}$ by the data processing inequality [8, pp. 34] we have

$$
I\left(\mathcal{P}\left(t_{i}\right) ; \mathcal{P}\left(t_{j}\right)\right) \geq I\left(\mathcal{P}\left(t_{i}\right) ; \mathcal{P}\left(t_{k}\right)\right)
$$

for all $i<j<k$. This suggests that estimates of mutual information between estimated device parameters can provide information on temporal ordering of the corresponding device parameters. Specifically, if $I\left(\mathcal{P}\left(t_{i}\right) ; \mathcal{P}\left(t_{j}\right)\right)<I\left(\mathcal{P}\left(t_{i}\right) ; \mathcal{P}\left(t_{k}\right)\right)$ then the time instant $t_{j}$ cannot lie between $t_{i}$ and $t_{k}$. This direct consequence of the data processing inequality suggests the following simple algorithm ${ }^{2}$ for temporal ordering of a set of estimated parameter sets $\mathcal{P}\left(t_{1}\right), \mathcal{P}\left(t_{2}\right), \ldots \mathcal{P}\left(t_{N}\right)$ :

$$
\begin{aligned}
& \text { 1. Choose any instant } t_{k} \text { and estimate the mutual information between the } \\
& \text { parameters at this instant and the parameters at all other time instants, i.e. } \\
& \text { obtain estimates }\left\{\hat{I}\left(\mathcal{P}\left(t_{k}\right) ; \mathcal{P}\left(t_{j}\right)\right)\right\}_{j=1}^{N} ; \\
& \text { 2. Select the time instant } t_{j_{0}} \text { that has the smallest mutual information value, } \\
& \text { viz., } \\
& \qquad j_{0}=\arg \min _{j} \hat{I}\left(\mathcal{P}\left(t_{k}\right) ; \mathcal{P}\left(t_{j}\right)\right)
\end{aligned}
$$

The time instant $t_{j_{0}}$ is temporally farthest from $t_{k}$ and must be at one end of the temporal range ;

3. Compute mutual information estimates between the parameters at the time instant $t_{j_{0}}$ and the parameters at all other time instants, i.e. obtain estimates $\left\{\hat{I}\left(\mathcal{P}\left(t_{j_{0}}\right) ; \mathcal{P}\left(t_{j}\right)\right)\right\}_{j=1}^{N}$;

4. Sort the mutual information estimated obtained in the previous step. This is the estimated temporal ordering.

Algorithm 1: Temporal Ordering Algorithm

In terms of running time, excluding the effort involved in estimating the mutual information, all steps except the sorting require time linear in $N$, whereas the sorting requires $O(N \log (N))$ time. Note also that the algorithm cannot resolve the direction of temporal ordering.

If the device parameters $\mathcal{P}(t)$ form a vector of independent identically distributed i.i.d. Gaussian processes, i.e.

$$
\mathcal{P}(t)=\left[V_{1}(t), V_{2}(t), \ldots V_{J}(t)\right]
$$

where $\left\{V_{n}(t)\right\}_{n=1}^{J}$ are independent Gaussian processes each having identical statistics, i.e., the same mean $\mu(t)=E\left[V_{n}(t)\right], n=$ $1,2, \ldots J$ and identical auto-covariance functions $C\left(t_{1}, t_{2}\right)=$ $E\left[\left(V_{n}\left(t_{1}\right)-\mu\left(t_{1}\right)\right)\left(V_{n}\left(t_{2}\right)-\mu\left(t_{2}\right)\right)\right], \forall n$. Then, we can readily relate the mutual information to the (common) auto-covariance function as follows [8]:

$$
\begin{aligned}
I\left(\mathcal{P}\left(t_{i}\right) ; \mathcal{P}\left(t_{j}\right)\right) & =\sum_{n=1}^{J} I\left(V_{n}\left(t_{i}\right) ; V_{n}\left(t_{j}\right)\right) \\
& =-\frac{J}{2} \log \left(1-\rho\left(t_{1}, t_{2}\right)^{2}\right)
\end{aligned}
$$

where $\rho\left(t_{1}, t_{2}\right)=C\left(t_{1}, t_{2}\right) / \sqrt{C\left(t_{1}, t_{1}\right) C\left(t_{2}, t_{2}\right)}$ is the correlation coefficient between times $t_{i}$ and $t_{j}$ for each of the component processes in $\mathcal{P}(t)$ indicated in (4). In this case, we see that the mutual information $I\left(\mathcal{P}\left(t_{i}\right) ; \mathcal{P}\left(t_{j}\right)\right)$ is monotonically related to the correlation coefficient $\rho\left(t_{1}, t_{2}\right)$ and hence the data processing inequality applies directly to the correlation coefficients and estimates thereof can be used for performing the ordering in Algorithm 1. For the specific case of sensor PRNU, a zero-mean i.i.d. Gaussian processes model is particularly apposite for the individual PRNU parameters and has been partly validated in prior sensor forensics work [9], though again without incorporating the temporal evolution.

\section{PRNU-BASED TEMPORAL FORENSICS OF DIGITAL IMAGES: PROBLEMS AND CHALLENGES}

As a concrete realization of our general framework, next we consider temporal forensics using PRNU estimates. The temporal model for this scenario was formulated in (2). Using a set of $M$ images taken

${ }^{2}$ For simplicity, the algorithm is stated under the assumption of strict inequality in (3). 
at the time instant $t$, a maximum likelihood estimate $\hat{K}(t)$ for the PRNU at time $t$ is obtained as [1]:

$$
\hat{K}(t)=\left[\sum_{k=1}^{N}\left(I_{k}(t)-\hat{I}_{k}(t)\right) \cdot I_{k}(t)\right] \cdot / \sum_{k=1}^{N}\left(I_{k}(t) \cdot I_{k}(t)\right)
$$

where $\hat{I_{k}}$ is a denoised version of image $I_{k}$ and ./ denotes elementby-element division operation over the pixels.

As outlined in Section 3, an i.i.d. zero-mean Gaussian model is well-suited for the PRNU parameters and thus the mutual information between $K\left(t_{1}\right)$ and $K\left(t_{2}\right)$ can be monotonically related to the correlation coefficient $\rho\left(t_{1}, t_{2}\right)$, which in turn can be estimated by computing the correlation coefficient between the PRNUs over the image pixels

$$
\hat{\rho}\left(t_{1}, t_{2}\right)=\sum_{a=1}^{A} \sum_{b=1}^{B} K_{a b}\left(t_{1}\right) K_{a b}\left(t_{2}\right)
$$

where $a$ and $b$ index the 2-D image pixel locations of the $A \times B$ PRNU estimates.

By utilizing the estimated correlation coefficients in Algorithm 1, we can now obtain a temporal ordering for the PRNU estimates and by induction for the corresponding images. Note that this process provides a method for ordering groups/clusters of images, that are known to be captured by the camera in close temporal proximity. For several applications, these clusters/groupings may be determined based on image content (for example images taken at a birthday party or clearly identifiable as a series) or based on proximity of upload time. We refer to this problem as Image cluster ordering (ICO).

A variant of ICO, where temporal ordering of a set of individual images is desired, may often be important in practice. In this scenario, the difficulties of estimating PRNU's from single images rather severely debilitates our framework. While the PRNU can be computed in principle from single images (i.e. for $N=1$ ), we observed that there is strong content interference from dark or textured regions in this scenario. We also consider a second problem that makes progress toward the individual image ordering problem, which we refer to as Individual Image Placement Within An Ordered Set of Clusters (IIP). This problem involves placing an individual image within a set of clusters or groups of images that have been ordered correctly in time and may be useful when the clusters and individual images have been obtained from different sources with the grouping preserved in the former. For example, folders of benign camera images could be obtained from a seized disk and it may be desirable to arrange illicit images taken by the same camera and posted online within the timeline established by the benign images.

\section{EXPERIMENTAL RESULTS}

In order to evaluate the performance of temporal forensics framework based on PRNU, we generate a database from native resolution images available on the well-known image sharing website Flickr [13]. Our database contains 1486 images downloaded from 3 different users with a wide variety of content. There are three unique cameras a) Canon EOS D10, b) Nikon Coolpix 2100, and c) Sony DSC $\mathrm{H} 1$ corresponding uniquely to the three users.

\subsection{Image Cluster Ordering (ICO)}

Recall, the ICO problem considers time ordering of groups of images captured within close proximity. For this experiment, we grouped images captured during the same month within a cluster and obtain a PRNU estimate from the cluster.

We then calculate correlation coefficients of PRNU estimates of different clusters. Figure 2 shows the correlation results for Nikon Coolpix 2100. In the figure, the axes correspond to time index of extracted PRNU estimates of a cluster of images. The color bar listed next to the figure shows the estimated correlation coefficients between the PRNU for individual clusters. We also list the correlation results for the Canon camera in Table 1. From the figure and the table, by comparing the values within each of the rows (equivalently columns) one can readily infer that Algorithm 1 yields the correct ordering for the image clusters irrespective of the time instant chosen in the initial step 1 . Thus the framework can indeed correctly order the image clusters.

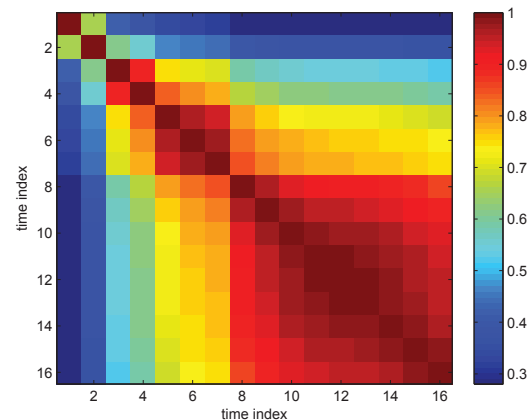

Fig. 2. Correlations for cluster of images that were captured a month apart from each other for Nikon Coolpix 2100. (See electronic version in color)

\subsection{Individual Image Placement Within An Ordered Cluster Set (IIP)}

We next consider the second problem described in Sec. 4 where it is desired to find the placement of an individual image relative to temporally ordered cluster set. In order to evaluate the performance of the proposed framework for this problem, we utilize the ordered cluster set from the first experiment (ICO).

The second problem (IIP) can be solved as follows. Given a set of clusters ordered temporally and an image, we calculate the PRNU signature of the image. We then calculate the mutual information of this PRNU with the PRNU estimates of each cluster. We estimate the timestamp of the image to be that of the cluster whose PRNU has the highest mutual information with the PRNU of the image. We conduct this experiment over 3 cameras and tabulate the results in Table 2. The table reveals that the proposed framework provides accurate solutions for the IIP problem.. A sample of the images for which the method provided inaccurate ordering are illustrated in Figure 3. As shown in the figure, most of the failed images (Figs. 3 (a)(b)) fall under the category of having highly textured or dark regions, and therefore, yield unreliable PRNU estimates. We also note that some of the failed images (in particular Fig. 3 (c)) were manipulated before being uploaded to Flickr.

\section{CONCLUSION AND DISCUSSION}

We pose a new problem of device temporal forensics and propose a novel two-stage framework to address this problem. By representing the temporal evolution of selected multimedia device parameters 


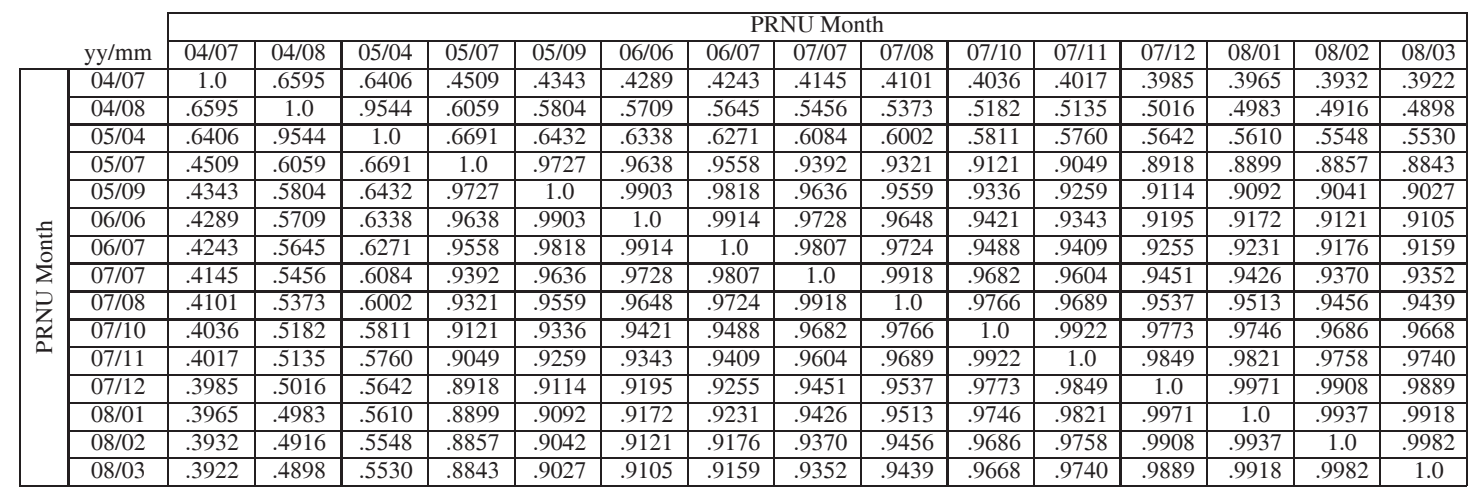

Table 1. Correlation coefficient values between 15 clusters of images captured by Canon EOS D10.

\begin{tabular}{c|c|c|c} 
& $\begin{array}{c}\text { Number of } \\
\text { Images }\end{array}$ & $\begin{array}{c}\text { Number of } \\
\text { Correct Ordering }\end{array}$ & $\begin{array}{c}\% \text { Correct } \\
\text { Ordering }\end{array}$ \\
\hline Canon & 102 & 87 & 85.3 \\
\hline Nikon & 42 & 38 & 90.59 \\
\hline Sony & 55 & 53 & 96.49
\end{tabular}

Table 2. Performance evaluation for ordering individual images on the timeline based on ordered cluster set.

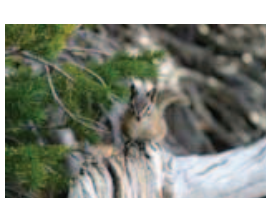

(a)

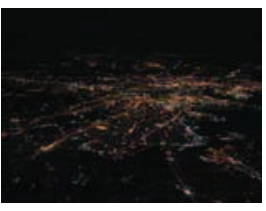

(b)

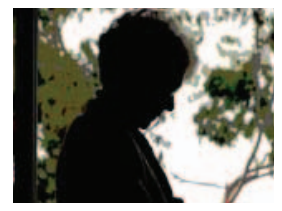

(c)
Fig. 3. Example images corresponding to inaccurate temporal ordering.

as a Markov process our framework enables temporal ordering by exploiting the data processing inequality in information theory. We demonstrate a specific realization of the framework for digital camera temporal forensics on photo-response non-uniformity (PRNU). Tests on a sampling of the Flickr website [13] indicate that the framework provides accurate temporal ordering for image clusters and accurate placement of individual images on an established timeline via temporal ordering of image clusters.

The effort presented here suggests several directions for further research - we highlight a couple of these. The PRNU provides an extremely high dimensional signal due to which the relatively simple Algorithm 1 performs well. Robust generalizations of this algorithm are, however, desirable that do not rely on a single "anchor" for establishing the timeline but instead utilize pair-wise estimates of mutual information. Alternate realizations of the framework are also of interest. In particular, we are currently exploring the application of the proposed framework to temporal forensics for printed images.

\section{ACKNOWLEDGMENT}

We thank Tomas Filler and Jessica Fridrich for sharing their database [14] of native resolution images from Flickr [13].

\section{REFERENCES}

[1] M. Chen, J. Fridrich, M. Goljan, and J. Lukas, "Determining image origin and integrity using sensor noise," IEEE Trans.
Info. Forensics and Security, vol. 3, no. 1, pp. 74-90, 2008.

[2] N. Khanna, G. T. C. Chiu, J. P. Allebach, and E. J. Delp, "Scanner identification with extension to forgery detection," in Proc. SPIE, vol. 6819, Jan. 2008, pp. 68 190G-68 190G.

[3] A. Swaminathan, M. Wu, and K. J. R. Liu, "Non-Intrusive Component Forensics of Visual Sensors Using Output Images," IEEE Trans. Info. Forensics and Security, vol. 2, no. 1, pp. 91-106, 2007.

[4] A. K. Mikkilineni, G. N. Ali, P. J. Chiang, G. T. Chiu, J. P. Allebach, and E. J. Delp, "Signature-embedding in printed documents for security and forensic applications," in Proc. SPIE, vol. 5306, Jan. 2004, pp. 455-466.

[5] O. Bulan, J. Mao, and G. Sharma, "Geometric distortion signatures for printer identification," in Proc. IEEE ICASSP, Apr. 2009 , accepted for presentation.

[6] S. Rekhis and N. Boudriga, "A Temporal Logic-Based Model for Forensic Investigation in Networked System Security," Lecture Notes In Computer Science, vol. 3685, p. 325, 2005.

[7] S. J. Stolfo, G. Creamer, and S. Hershkop, "A temporal based forensic analysis of electronic communication," in Proceedings of the 2006 international conference on Digital government research. ACM New York, NY, USA, 2006, pp. 23-24.

[8] T. Cover and J. A. Thomas, Elements of Information Theory, 2nd ed. New York: John Wiley and Sons, 2006.

[9] J. Lukas, J. Fridrich, and M. Goljan, "Digital camera identification from sensor pattern noise," IEEE Trans. Info. Forensics and Security, vol. 1, no. 2, pp. 205-214, 2006.

[10] A. E. Dirik, H. T. Sencar, and N. Memon, "Digital single lens reflex camera identification from traces of sensor dust," IEEE Trans. Info. Forensics and Security, vol. 3, no. 3, pp. 539-552, Sept. 2008.

[11] N. Khanna, A. K. Mikkilineni, G. T. C. Chiu, J. P. Allebach, E. J. Delp, et al., "Scanner Identification Using Sensor Pattern Noise," in Proc. SPIE, vol. 6505, Jan. 2007, pp. 6505-65 051K.

[12] A. Leon-Garcia, "Probability and Random Processes For EE's," 2007.

[13] "Flickr." [Online]. Available: http://www.flickr.com/

[14] T. Filler, J. Fridrich, and M. Goljan, "Using sensor pattern noise for camera model identification," in Proc. IEEE ICIP, Oct. 2008, pp. 1296-1299. 